\title{
Logarithmic Negativity: A Full Entanglement Monotone That is not Convex
}

\author{
M. B. Plenio \\ QOLS, Blackett Laboratory, Imperial College London, Prince Consort Road, London SW7 2BW, United Kingdom \\ Institute for Mathematical Sciences, Imperial College London, 53 Exhibition Road, London SW7 2BW, United Kingdom
}

(Received 16 May 2005; published 26 August 2005; corrected 1 September 2005)

\begin{abstract}
It is proven that logarithmic negativity does not increase on average under a general positive partial transpose preserving operation (a set of operations that incorporate local operations and classical communication as a subset) and, in the process, a further proof is provided that the negativity does not increase on average under the same set of operations. Given that the logarithmic negativity is not a convex function this result is surprising, as it is generally considered that convexity describes the local physical process of losing information. The role of convexity and, in particular, its relation (or lack thereof) to physical processes is discussed and importance of continuity in this context is stressed.
\end{abstract}

PACS numbers: 03.67.Mn, 03.67.Hk, 05.70.-a

Introduction. - Entanglement is the key resource in many quantum information processing protocols. Therefore it is of interest to develop a detailed understanding of its properties. In view of the resource character of entanglement it is of particular interest to be able to quantify entanglement [1-5].

Any resource is intimately related to a constraint which the resource allows us to overcome. Therefore, the detailed character of entanglement and its quantification as a resource depends on the constraints that are being imposed on the set of operations. In a communication setting where two spatially separated parties aim to manipulate a joint quantum state it is natural to restrict attention to local quantum operations and classical communication (LOCC). In this case separable states are freely available while nonseparable states, which cannot be prepared by LOCC alone, become a resource. The phenomenon of bound entanglement [6], i.e., nonseparable states that possess a positive partial transpose and are useless to distill pure maximally entangled states by LOCC $[7,8]$, suggest that there are other natural restricted classes of operations. One might add bound entangled states as a free resource to LOCC operations to achieve tasks that are impossible under LOCC alone [9-11]. Encompassing both classes is the mathematically more natural and convenient class of positive partial transpose preserving operations (PPT-operations) [12] which have the property that they map the set of positive partial transpose states into itself just as LOCC operations map the set of separable states into itself. Under this set of operations distillable quantum states become a valuable resource while bound entanglement is free.

A function $E$ that is suggested to quantify entanglement must satisfy certain conditions. Apart from the requirement that $E$ vanish on the set of states that can be created using LOCC (or PPT) alone, the most important property is that of the nonincrease on average of $E$ under LOCC (or PPT) [1-5], i.e.,

$$
E(\rho) \geq \sum_{i} p_{i} E\left(\rho_{i}\right)
$$

where, in a LOCC (PPT) protocol applied to state $\rho$, the state $\rho_{i}$ with label $i$ is obtained with probability $p_{i}$ [see part (a) of Fig. 1 for an illustration]. Note that Eq. (1) is more restrictive than the requirement that entanglement decreases under the less general set of operations implementing $\rho \rightarrow \sigma=\sum_{i} p_{i} \rho_{i}$ [see part (b) of Fig. 1], i.e., that $E(\rho) \geq E\left(\sum_{i} p_{i} \rho_{i}\right)$. Restricting attention to such operations would imply an additional constraint, namely, that we are unable to select subensembles according to a measurement outcome. Such an additional constraint is not directly related to the nonlocal structure of quantum mechanics and would obscure key features of entanglement. Therefore we consider here, as in the bulk of the literature, condition Eq. (1).

A quantity $E$ possessing the above properties, in particular, Eq. (1), is called an entanglement monotone. If such a monotone is furthermore identical to the entropy of entanglement on pure states [13], i.e., the entropy of the reduced density operator of one party, then it is called an entanglement measure [5].

A significant number of such entanglement monotones and entanglement measures have been formulated and their properties have been explored [13-31]. While many of these quantities, for example, the entanglement cost and the distillable entanglement, are operationally motivated, their mathematical formulation generally involves an opti-

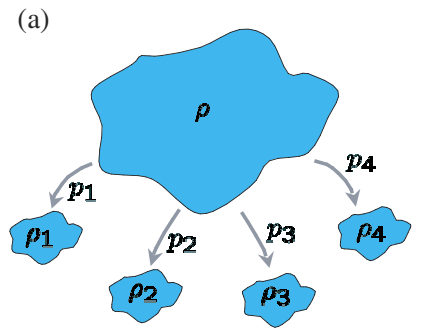

(b)

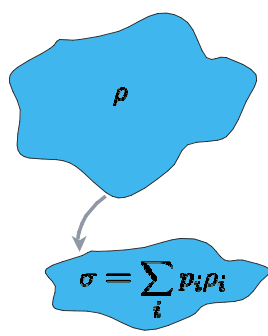

FIG. 1 (color online). Schematic picture of the action of quantum operations with and without subselection shown in part (a) and part (b), respectively. 
mization over high-dimensional spaces which makes their evaluation exceedingly difficult. Even when the minimization involves a convex function on a convex set $[15,16]$ so that the optimization is numerically feasible, analytical expressions are generally not available except in cases of very high symmetry [32,33].

It is therefore of some interest to find quantities that are entanglement monotones, i.e., satisfy Eq. (1) and are at the same time easy to compute. Such quantities are valuable even if for pure states they do not coincide with the entropy of entanglement and/or lack an operational interpretation. One example is the so-called negativity [34-36], to be defined below, which has been proven to be an entanglement monotone [28,37-39]. Unfortunately, the negativity does not possess a striking operational interpretation. Its close cousin the logarithmic negativity, on the other hand, is an upper bound to distillable entanglement and has an operational interpretation [11]. The logarithmic negativity is, however, not convex. This implies that it can increase under mixing, a process which is often considered to describe the loss of classical information, i.e., a local process. Therefore it was believed that the logarithmic negativity is not an entanglement monotone in the sense of Eq. (1) (see, for example, page 3 of [28]). However, this reasoning is not conclusive and, in fact, incorrect.

Following some basic definitions, we discuss the relation of convexity to physical processes in general and the loss of classical information and highlight the importance of continuity in this context. Then, we proceed to present a proof that demonstrates that indeed both negativity and the logarithmic negativity are entanglement monotones, satisfying Eq. (1), both under LOCC and under PPT operations.

Notations and definitions. - For any operator $A$ we define the trace norm $\|A\|_{1}=\operatorname{tr}|A|=\operatorname{tr} \sqrt{A^{\dagger} A}$, i.e., the sum of the singular values of $A[40,41]$. Employing the trace norm we then define the negativity as

$$
N(\rho)=\frac{\left\|\rho^{\Gamma_{A}}\right\|_{1}-1}{2}
$$

where $\rho^{\Gamma_{A}}$ (in the following we will drop the index $A$ ) denotes the partial transpose of $\rho$ with respect to party $A$. This definition ensures that the negativity vanishes on PPT states and coincides with the entropy of entanglement on maximally entangled states. Note that the negativity differs from the entropy of entanglement for all other pure entangled states. It is known that the negativity is an entanglement monotone under general LOCC operations as well as PPT operations in the sense of Eq. (1) [28,37-39].

A more easily interpreted and useful quantity is obtained by considering the logarithmic negativity [34] which is defined by

$$
L N(\rho)=\log _{2}\left\|\rho^{\Gamma}\right\|_{1} .
$$

This quantity exhibits monotonic behavior under LOCC and PPT operations $\Psi$ in the sense $L N(\Psi(\sigma)) \leq L N(\sigma)$, i.e., in processes not involving subselection. It is an upper bound to distillable entanglement [28] and possesses an operational interpretation as a special type of entanglement cost under PPT operations [11]. For general LOCC or PPT operations it is not known, however, whether the logarithmic negativity is an entanglement monotone, i.e., whether Eq. (1) is satisfied. Indeed, it was suggested (see, for example, page 3 of [28]) that the lack of convexity of the logarithmic negativity implies the existence of LOCC operations that increase the logarithmic negativity on average. We will now discuss why the lack of convexity alone is not sufficient to destroy monotonicity in the sense of Eq. (1), highlight the importance of continuity, and then present a rigorous proof for the monotonicity of both the negativity and the logarithmic negativity.

Convexity issues. - In the present context it is important to note that the convexity requirement itself is not straightforwardly connected to the physical process of discarding of information [5,32]. The loss of information refers to a situation where one begins with a selection of locally identifiable states $\rho_{i}$ that appear with rate $p_{i}$ to end up in a mixture of these states which is of the form $\rho=\sum p_{i} \rho_{i}$. Indeed, the first situation, before the loss of information about the state, can be described by the quantum state

$$
\sum_{i} p_{i}|i\rangle_{M}\langle i| \otimes \rho_{i}^{A B},
$$

where $\left\{|i\rangle_{M}\right\}$ denote some orthonormal product basis. Clearly a measurement of the marker particle $M$ reveals the identity of the state of parties $A$ and $B$ without disturbing the associated states $\rho_{i}$. The loss of information about the identity of the states $\rho_{i}$ is then described by tracing out the marker particle $M$ to obtain $\rho=\sum p_{i} \rho_{i}[5,42]$. This process should not increase entanglement and we would like to see that

$$
E\left(\sum_{i} p_{i}|i\rangle_{M}\langle i| \otimes \rho_{i}^{A B}\right) \geq E(\rho)
$$

is satisfied. Indeed, this requirement is a special case of Eq. (1). It is important to note, however, that this process is not identical to the mathematically convenient convexity requirement

$$
\sum_{i} p_{i} E\left(\rho_{i}\right) \geq E\left(\sum_{i} p_{i} \rho_{i}\right) .
$$

Indeed, one can explicitly demonstrate that the logarithmic negativity is a concave function on Werner states and can therefore increase under mixing of quantum states [11]. One might argue, however, that there is a connection between mixing and the loss of information in the asymptotic limit. Asymptotic mixing, i.e., the process

$$
\otimes_{i=1}^{k} \rho_{1}^{\otimes p_{i} N} \rightarrow\left(\sum_{i=1}^{k} p_{i} \rho_{i}\right)^{\otimes N}
$$

can be realized in the limit $N \rightarrow \infty$ with arbitrary precision using only LOCC [43]. This appears to suggest that mixing 
and the loss of information are identical but one should note that this is only so for quantities that possess sufficiently strong continuity properties in the asymptotic limit. This is not obviously so for the logarithmic negativity as is already suggested by the lockability of the logarithmic negativity [44]. As the detailed continuity properties of the logarithmic negativity are not known we are not able to connect the convexity with local loss of information here. Therefore the monotonicity of the logarithmic negativity is an open question that needs to be settled directly.

Monotonicity properties. - We will now prove that the logarithmic negativity as well as the negativity are entanglement monotones in the sense of Eq. (1) both under general LOCC operations as well as the more general PPT operations.

Lemma. - The logarithmic negativity is an entanglement monotone, satisfying Eq. (1), for general trace-preserving completely positive PPT operations.

Proof.-The proof proceeds in two steps. First we consider the monotonicity properties of $\left\|\rho^{\Gamma}\right\|_{1}$. For tracepreserving completely positive PPT operations (denoted CP-PPT) this will exhibit the same behavior as the negativity itself [45]. Then we will proceed to demonstrate that this implies the monotonicity of the logarithmic negativity.

Let us consider a completely positive PPT operation $\Psi$ that maps $\rho$ to $\sigma=\Psi(\rho)$ deterministically and denote with $A_{+}\left(A_{-}\right)$the positive (negative) part of the operator $A$. Employing the linearity of $\Psi$ and the fact that $\Psi$ maps positive states to positive states, we find

$$
\begin{aligned}
\operatorname{tr}|\Psi(\rho)| & =\operatorname{tr}\left\{\Psi(\rho)_{+}-\Psi(\rho)_{-}\right\} \\
& =\operatorname{tr}\left\{\Psi(\rho)_{+}+\Psi(-\rho)_{+}\right\} \\
& \leq \operatorname{tr}\left\{\Psi\left(\rho_{+}\right)\right\}+\operatorname{tr}\left\{\Psi\left((-\rho)_{+}\right)\right\} \\
& =\operatorname{tr}\left\{\Psi\left(\rho_{+}\right)\right\}+\operatorname{tr}\left\{\Psi\left(-(\rho)_{-}\right)\right\} \\
& =\operatorname{tr}\left\{\Psi\left(\rho_{+}-\rho_{-}\right)\right\} \\
& =\operatorname{tr} \Psi(|\rho|)
\end{aligned}
$$

Now consider a general CP-PPT operation that maps $\rho$ to $\rho_{i}=\Psi_{i}(\rho) / \operatorname{tr} \Psi_{i}(\rho)$ with probability $p_{i}=\operatorname{tr} \Psi_{i}(\rho)$ such that all the $\Psi_{i}$ are CP-PPT maps and $\Sigma_{i} \Psi_{i}$ is trace preserving. Employing Eq. (7) we find

$$
\begin{aligned}
\sum_{i} p_{i}|| \rho_{i}^{\Gamma} \|_{1} & =\sum_{i} \operatorname{tr}\left|\left(\Psi_{i}(\rho)\right)^{\Gamma}\right| \\
& =\sum_{i} \operatorname{tr}\left|\Psi_{i}^{\Gamma}\left(\rho^{\Gamma}\right)\right| \\
& \leq \sum_{i} \operatorname{tr} \Psi_{i}^{\Gamma}\left(\left|\rho^{\Gamma}\right|\right) \\
& =\sum_{i} \operatorname{tr}\left(\Psi_{i}\left(\left|\rho^{\Gamma}\right|^{\Gamma}\right)\right)^{\Gamma} \\
& =\operatorname{tr}\left|\rho^{\Gamma}\right|^{\Gamma} \\
& =\left\|\rho^{\Gamma}\right\|_{1} .
\end{aligned}
$$

This demonstrates the monotonicity of $\left\|\rho^{\Gamma}\right\|_{1}=\operatorname{tr}\left|\rho^{\Gamma}\right|$.
To prove the monotonicity, in the sense of Eq. (1), of the logarithmic negativity we use Eq. (8), the concavity of the logarithm, and its monotonicity to obtain

$$
\begin{aligned}
\sum_{i} p_{i} L N\left(\rho_{i}\right) & =\sum_{i} p_{i} \log _{2}\left\|\rho_{i}^{\Gamma}\right\|_{1} \\
& \leq \log _{2} \sum_{i} p_{i}\left\|\rho_{i}^{\Gamma}\right\|_{1} \\
& \leq \log _{2}\left\|\rho^{\Gamma}\right\|_{1} \\
& =L N(\rho) .
\end{aligned}
$$

This is the monotonicity of the logarithmic negativity under general PPT operations in the sense of Eq. (1) which completes the proof.

Summary and conclusions. - We proved that the negativity is an entanglement monotone both under LOCC, for which we also provide an alternative, previously unpublished proof [39] complementing existing proofs $[28,37,38]$, and under the more general setting of positive partial transpose preserving operation. We extended this result further to also prove that the logarithmic negativity, which possesses an operational interpretation [11], is an entanglement monotone both under general LOCC and PPT operations. This is despite the logarithmic negativity being neither convex nor concave, a fact that has previously led to the expectation that the logarithmic negativity cannot be a full entanglement monotone. The key observation, however, is that convexity is merely a mathematical requirement for entanglement monotones and generally does not correspond to a physical process describing the loss of information about a quantum system. Indeed, it is the concavity in combination with the monotonicity of the logarithm that permits the proof of the nonincrease of the logarithmic negativity under PPT operations.

The author is grateful for discussions with K. Audenaert, D. Browne, J. Eisert, S. Ishizaka, S. Virmani, and R.F. Werner on the subject of this Letter. Careful reading and encouraging comments by M. Christandl are gratefully acknowledged. This work is part of the QIP-IRC (www. qipirc.org) supported by EPSRC (GR/S82176/0) as well as the EU Thematic Network QUPRODIS (IST-2001-38877) and the Leverhulme Trust (F/07 058/U).

[1] M. B. Plenio and V. Vedral, Contemp. Phys. 39, 431 (1998).

[2] M. Horodecki, Quantum Inf. Comput. 1, 3 (2001).

[3] P. Horodecki and R. Horodecki, Quantum Inf. Comput. 1, 45 (2001).

[4] J. Eisert and M. B. Plenio, Int. J. Quantum. Inform. 1, 479 (2003).

[5] M. B. Plenio and S. Virmani, quant-ph/0504163.

[6] M. Horodecki, P. Horodecki, and R. Horodecki, Phys. Rev. Lett. 80, 5239 (1998).

[7] A. Peres, Phys. Rev. Lett. 77, 1413 (1996).

[8] M. Horodecki, P. Horodecki, and R. Horodecki, Phys. Lett. A 223, 1 (1996). 
[9] S. Ishizaka, Phys. Rev. Lett. 93, 190501 (2004).

[10] S. Ishizaka and M. B. Plenio, Phys. Rev. A 71, 052303 (2005).

[11] K. Audenaert, M. B. Plenio, and J. Eisert, Phys. Rev. Lett. 90, 027901 (2003).

[12] E. M. Rains, IEEE Trans. Inf. Theory 47, 2921 (2001).

[13] C.H. Bennett, H. Bernstein, S. Popescu, and B. Schumacher, Phys. Rev. A 53, 2046 (1996).

[14] C. H. Bennett, D. P. DiVincenzo, J. A. Smolin, and W. K. Wootters, Phys. Rev. A 54, 3824 (1996).

[15] V. Vedral and M. B. Plenio, Phys. Rev. A 57, 1619 (1998).

[16] V. Vedral, M. B. Plenio, M. A. Rippin, and P. L. Knight, Phys. Rev. Lett. 78, 2275 (1997).

[17] M. Horodecki, quant-ph/0412210.

[18] W. K. Wootters, Phys. Rev. Lett. 80, 2245 (1998).

[19] M. B. Plenio, S. Virmani, and P. Papadopoulos, J. Phys. A 33, L193 (2000).

[20] M. Horodecki, P. Horodecki, and R. Horodecki, Phys. Rev. Lett. 84, 2014 (2000).

[21] S. Virmani and M. B. Plenio, Phys. Lett. A 268, 31 (2000).

[22] K. Audenaert, J. Eisert, E. Jané, M. B. Plenio, S. Virmani, and B. DeMoor, Phys. Rev. Lett. 87, 217902 (2001).

[23] M. B. Plenio and V. Vedral, J. Phys. A 34, 6997 (2001).

[24] P. Hayden, M. Horodecki, and B. M. Terhal, J. Phys. A 34, 6891 (2001).

[25] K. Audenaert, B. DeMoor, K. G. H. Vollbrecht, and R. F. Werner, Phys. Rev. A 66, 032310 (2002).

[26] M. J. Donald, M. Horodecki, and O. Rudolph, J. Math. Phys. (N.Y.) 43, 4252 (2002).

[27] R. R. Tucci, quant-ph/0202144.

[28] G. Vidal and R. F. Werner, Phys. Rev. A 65, 32314 (2002).

[29] J. Eisert, K. Audenaert, and M. B. Plenio, J. Phys. A 36, 5605 (2003).

[30] M. Christandl and A. Winter, J. Math. Phys. (N.Y.) 45, 829 (2004).

[31] M. Horodecki, P. Horodecki, R. Horodecki, J. Oppenheim, A. Sen(De), U. Sen, and B. Synak, Phys. Rev. A 71, 062307 (2005).

[32] J. Eisert, T. Felbinger, P. Papadopoulos, M. B. Plenio, and M. Wilkens, Phys. Rev. Lett. 84, 1611 (2000).

[33] K. G. H. Vollbrecht and R.F. Werner, Phys. Rev. A 64, 062307 (2001).

[34] R.F. Werner (private communication).

[35] K. Zyczkowski, P. Horodecki, A. Sanpera, and M. Lewenstein, Phys. Rev. A 58, 883 (1998).

[36] J. Eisert and M. B. Plenio, J. Mod. Opt. 46, 145 (1999).

[37] J. Lee, M. S. Kim, Y. J. Park, and S. Lee, J. Mod. Opt. 47, 2151 (2000).

[38] J. Eisert, Ph.D. thesis, University of Potsdam, 2001.

[39] Following the announcement of a proof for the monotonicity of the negativity by J. Eisert (later published in [38]), M.B.P. constructed the following previously unpublished proof (referred to as Ref. [39] in [28]). Any
LOCC operation can be constructed from four basic operations (i) Appending a local ancilla in a pure state, (ii) Application of unilocal unitary transformations, (iii) Local projective measurements on the ancillas and subselection, (iv) Tracing out of ancillas. We will now show that the negativity does not increase under any of these operations: (i) The eigenvalues of $\rho^{\Gamma}$ are the same as those of $(|0\rangle\langle 0| \otimes \rho)^{\Gamma}$. (ii) Local unitaries leave the spectrum of $\rho^{\Gamma}$ invariant. (iii) The trace norm is unitarily invariant and for any set of orthogonal projectors and any unitarily invariant norm $\||\cdot \||$ we have $\|\left|\sum_{i} P_{i} A P_{i} \|\right| \leq$ ||A||| [40]. Then, for mutually orthogonal local projectors $\left\{P_{i}^{A}\right\}$ and $\left\{P_{i}^{B}\right\}$

$$
\begin{aligned}
\left\|\rho^{\Gamma}\right\|_{1} & \geq\left\|\sum_{i} P_{i}^{A} \otimes\left(P_{i}^{B}\right)^{\Gamma} \rho^{\Gamma} P_{i}^{A} \otimes\left(P_{i}^{B}\right)^{\Gamma}\right\|_{1} \\
& =\left\|\left(\sum_{i} P_{i}^{A} \otimes P_{i}^{B} \rho P_{i}^{A} \otimes P_{i}^{B}\right)^{\Gamma}\right\|_{1} \\
& =\sum_{i}\left\|\left(P_{i}^{A} \otimes P_{i}^{B} \rho P_{i}^{A} \otimes P_{i}^{B}\right)^{\Gamma}\right\|_{1} .
\end{aligned}
$$

(iv) Tracing out an ancilla can be viewed as first measuring it in some orthonormal basis without subselection and then tracing the ancilla. Making use of $\|A+B\|_{1} \leq\|A\|_{1}+$ $\|B\|_{1}[40]$, and ancilla system $C$ and one-dimensional projectors $P_{i}^{C}$ we find

$$
\begin{aligned}
\left\|\rho_{(C A) B}^{\Gamma}\right\|_{1} & \geq\left\|\sum_{i} P_{i}^{C} \otimes \mathbf{1} \rho_{(C A) B}^{\Gamma} P_{i}^{C} \otimes \mathbf{1}\right\|_{1} \\
& =\left\|\sum_{i} P_{1}^{C} \otimes \operatorname{tr}_{C}\left\{P_{i}^{C} \rho_{i}^{\Gamma} P_{i}^{C}\right\}\right\|_{1} \\
& =\sum_{i}\left\|\operatorname{tr}_{C}\left\{P_{i}^{C} \rho_{i}^{\Gamma} P_{i}^{C}\right\}\right\|_{1} \\
& \geq\left\|\sum_{i} \operatorname{tr}_{C}\left\{P_{i}^{C} \rho_{i}^{\Gamma} P_{i}^{C}\right\}\right\|_{1} \\
& =\left\|\left(\operatorname{tr}_{C} \rho_{(A C) B}\right)^{\Gamma}\right\|_{1} .
\end{aligned}
$$

In conclusion, we have shown that $\left\|\rho^{\Gamma}\right\|_{1}$ and therefore the negativity does not increase under (i) -(iv) and therefore also not under LOCC operations.

[40] R. Bhatia, Matrix Analysis (Springer, Heidelberg, 1997).

[41] R. A. Horn and C.R. Johnson, Matrix Analysis (Cambridge University Press, Cambridge, 1987).

[42] M. B. Plenio and V. Vitelli, Contemp. Phys. 42, 25 (2001).

[43] G. Vidal, quant-ph/0203107.

[44] K. Horodecki, M. Horodecki, P. Horodecki, and J. Oppenheim, Phys. Rev. Lett. 94, 200501 (2005).

[45] For non-trace-preserving CP-PPT operations this is, however, unclear although results in [10] suggest that monotonicity of the negativity might hold even under non-tracepreserving PPT operations. 